Cinémas

Revue d'études cinématographiques

Journal of Film Studies

\title{
Martin Barnier, Bruits, cris, musiques de films. Les projections avant 1914, Rennes, Presses Universitaires de Rennes, 2010
}

\section{Germain Lacasse}

Volume 22, numéro 1, automne 2011

URI : https://id.erudit.org/iderudit/1005809ar

DOI : https://doi.org/10.7202/1005809ar

Aller au sommaire du numéro

Éditeur(s)

Cinémas

ISSN

1181-6945 (imprimé)

1705-6500 (numérique)

Découvrir la revue

Citer ce compte rendu

Lacasse, G. (2011). Compte rendu de [Martin Barnier, Bruits, cris, musiques de films. Les projections avant 1914, Rennes, Presses Universitaires de Rennes, 2010]. Cinémas, 22(1), 177-181. https://doi.org/10.7202/1005809ar d'utilisation que vous pouvez consulter en ligne.

https://apropos.erudit.org/fr/usagers/politique-dutilisation/ 
Martin Barnier, Bruits, cris, musiques de films. Les projections avant 1914, Rennes, Presses Universitaires de Rennes, 2010.

Sur la place du village est arrivé le "Grand Cirque Électrique Moderne Barnier». La plus grande tente est celle du "Cynématographe ", qu'on remarque par sa devanture colorée mais surtout par les bruits divers qui l'entourent. Un tracteur à vapeur roule sans cesse pour produire l'électricité nécessaire au fonctionnement du "Cynématographe», un bonisseur crie à tue-tête pour attirer le public, et sous la tente le spectateur regarde les films en applaudissant ou en huant les pitreries qu'on lui offre, pendant que les serveurs crient pour recouvrir la musique reproduite par le gramophone, qui les empêche de bien entendre les commandes des clients. Comme le «Grand Cirque..." s'est installé tout juste entre la gare et le marché public, le bruit des trains et les cris du marché viennent s'ajouter au tintamarre qui entoure les projections. Heureusement que les films sont «muets»!

Cette assourdissante description des premières projections de films n'est pas une citation, elle est reconstituée à partir du livre de Martin Barnier Bruits, cris, musiques de films. Les projections avant 1914. Plusieurs études importantes ont été consacrées depuis deux décennies au son du cinéma "muet» (Altman, Pisano, Boillat, etc.), mais celle-ci l'aborde d'une façon inattendue quoique très pertinente: au lieu de s'arrêter aux sons destinés à l'accompagnement du film muet, elle inventorie et étudie de façon exhaustive tout l'environnement sonore des premières projections, y compris tous les bruits qui n'ont rien à voir avec le film mais font partie à l'époque de son paysage sonore. Ce livre est aussi stimulant par ses propositions théoriques que par la diversité et l'originalité de sa documentation et suscite la 
réflexion sur des sujets et des hypothèses inouïes, c'est le cas de le dire.

Barnier explique et justifie l'intérêt de sa démarche:

Dans le cadre de ce travail nous essaierons de construire une «histoire du sensible». En nous méfiant de l'aspect «oculocentriste" de la société occidentale nous saisirons les affects et les sensations. [...] Essayons de retrouver les sensations auditives des publics de la Belle Époque, pour comprendre ce qu'était la projection de films. De cette façon, nous pourrons expliquer la "spectacularisation" de la vie de la fin du XIX siècle et l'émergence d'une culture de masse» (p. 25).

Cette proposition, qu'il présente dans son introduction, est complétée par une autre aussi importante dans sa conclusion:

Les observations des manifestations bruyantes du public, dans un environnement très sonore, nous montrent un monde cinématographique éloigné des considérations alarmistes d'Adorno, Horkheimer et les théories de "manipulation des foules» de l'école de Francfort. [...] Au contraire, l'aspect vivant et turbulent des séances de la Belle Époque montre un spectateur émancipé. Le spectateur contrôle parfois la séance et influence souvent les « organisateurs» (p. 269).

Martin Barnier nous propose donc une étude détaillée, méthodique et rigoureuse mais destinée aussi à mieux fonder théoriquement les approches de l'histoire du son au cinéma. Le livre est d'ailleurs préfacé par Rick Altman, dont le colossal ouvrage (2004) a déjà bien montré la pertinence d'une histoire sonore des débuts du cinéma par une approche performative polysémique qu'il a décrite à l'aide de l'expression «sound as event». Le livre de Martin Barnier s'inscrit dans cette approche en y ajoutant en quelque sorte un volet phénoménologique fondé sur les sensations multiples vécues par les spectateurs, mais basé aussi sur l'inventaire et l'étude de leurs «réactions sonores». Son histoire comporte aussi un aspect sociologique, montrant non seulement que les sons expriment les réponses des différents publics mais aussi les variétés de leurs réactions selon les classes sociales et les régions, certains auditoires étant aussi réservés que d'autres sont bruyants. 
Très court, le premier chapitre traite des sonorités entourant les projections avant l'arrivée des images animées: "harmonica de verre" avec les fantasmagories de Robertson, chansons avec les bandes d'Émile Reynaud, bruits d'eau pendant les spectacles d'ombre, etc. Après cette entrée en matière prometteuse, le deuxième chapitre offre la partie sans doute la plus originale de l'ouvrage: "Vacarmes de foire». Le lecteur peut entendre ici la plus surprenante et hétéroclite évocation des sensations sonores offertes aux spectateurs de la Belle Époque, dans les théâtres chics ou les foires de province: musiciens, chanteurs, phonographes, pianos mécaniques, crieurs, bonimenteurs, conférenciers, mais Barnier lui fait surtout entendre les autres sources de bruits, toujours oubliées dans les études sur le son: machines à vapeur, bruits de ville et de foule, applaudissements et chahut, murmures et conversations, cris et acclamations, bruits de vaisselle et de buvette, auxquels s'ajoutent même des cris d'animaux de cirque dans une attraction baptisée "Zoo-Cinéma» (p. 56). Ce chapitre illustre mieux l'originalité du travail de Barnier: il est carrément sorti du paradigme "son du film» pour intégrer à son étude toutes les occurrences sonores entourant les différentes pratiques de projection. En élargissant ainsi la perspective sur le son, il raffine considérablement la compréhension des spectacles de cette époque où les films étaient souvent des attractions parmi d'autres, présentés dans des lieux aussi hétéroclites que les spectacles.

Le troisième chapitre, "Cris, applaudissements et bruits du public», associe justement les réactions diverses des spectateurs avec les caractéristiques des lieux et des auditoires: silences polis et applaudissements dans les salles huppées, tintamarre dans les projections ayant lieu dans des foires et dans des bars, "émotions et bagarres» (p. 72), cris et jeux d'enfants, etc. Ici se trouve peutêtre la plus importante information sur l'autre versant de l'étude du son: la réaction des spectateurs. Ici non plus l'auteur ne s'arrête pas à ce qui répond directement à l'écoute du film, il déborde la question et s'attarde à toutes les autres réactions, individuelles ou collectives, sortant ainsi de l'habituel cadre qui limite la documentation et l'étude à ce qui concerne uniquement le rapport étroit avec l'œuvre. Barnier dépasse ce modèle et 
développe une approche contextuelle des pratiques qui permet de saisir celles-ci selon les paramètres de leur époque plutôt que selon les conceptions ultérieures. Il poursuit ce travail raffiné dans le chapitre 4, portant sur les conférenciers et bonimenteurs. Son travail n'ajoute pas beaucoup de documentation à ce que l'on sait sur ces artistes en France, mais il raffine la description de leur pratique et la théorie qui l'entoure en distinguant quelles sortes de fonctions ils exercent selon les lieux où ils travaillent et quels sont les modèles qu'ils se donnent eux-mêmes (manuels pour conférenciers, conseils entre collègues, etc.). Compte tenu de l'angle choisi, Barnier innove surtout en décrivant l'ambiance sonore dans laquelle doivent s'exprimer les différents aboyeurs, conférenciers, commentateurs, narrateurs, dont la voix doit maintenant être entendue dans le vacarme plutôt qu'entourée de silence. Il s'agit d'une contribution importante aux trop rares études sur ces artisans oubliés du cinéma muet avec guillemets.

Les chapitres suivants sont consacrés aux interventions sonores plus souvent associées au son du cinéma muet: bruitages, musiques, synchronisations. Ici encore l'ouvrage approfondit ce que l'on sait déjà au sujet du matériel utilisé mais aussi sur la diversité de ses utilisations selon les régions et les salles: machines à bruits, bruitage «à la bouche» (p. 135), musiques préparées ou improvisées, chanteurs populaires ou artistes lyriques. La partie sur les «synchronisations» (p. 189-257, le plus long chapitre) est la plus documentée, mais elle est aussi importante par son aspect historiographique. La très grande diversité des sources qu'il a explorées et la minutie de sa réflexion permettent à Barnier de démontrer que les projections synchronisées ont été beaucoup plus nombreuses et durables que le croyaient les historiens: «Des centaines de films parlants et chantants ont été vus et entendus par les spectateurs français pendant la Belle Époque»(p. 189). Il approfondit ce qui est déjà connu sur les Phonoscènes Gaumont, mais il montre aussi que la firme Pathé a développé et diffusé cette sorte de films de façon intense et pendant très longtemps. Auteur d'un ouvrage antérieur sur l'arrivée du son au cinéma, Barnier souligne dans son nouvel ouvrage que la sonorisation était beaucoup plus fréquente que l'on pense, même pendant la période qu'il étudie ici. 
Dans sa brève conclusion, Barnier revient sur les concepts qui ont orienté sa recherche. Il ne développe pas outre mesure ces considérations, mais il nous laisse comprendre clairement que l'histoire qu'il a exposée est conçue: s'il a élargi son écoute à tout ce qui entoure le spectateur sans concerner directement le film, c'est qu'il croit à une approche contextuelle où l'historien essaie de se mettre en phase avec les concepts de l'époque étudiée, plutôt que de rester campé sur sa vision a posteriori de spécialiste. C'est aussi qu'il croit en ce que Jacques Rancière appelle «le spectateur émancipé» (2008) et qu'ainsi il essaie de comprendre l'intelligence du spectateur de la Belle Époque, peu importe qu'il soit dans une salle de foire ou dans un théâtre des grands boulevards. La qualité de la documentation, de son interprétation et de l'approche historique exposée dans ce livre force l'admiration des lecteurs autant que des collègues historiens. "Ouvrage majeur dans son champ" ne serait pas un qualificatif exagéré.

Germain Lacasse

Université de Montréal

\section{RÉFÉRENCES BIBLIOGRAPHIQUES}

Altman 2004: Rick Altman, Silent Film Sound, New York, Columbia University Press, 2004.

Boillat 2007: Alain Boillat, Du bonimenteur à la voix-over. Voix-attraction et voixnarration au cinéma, Lausanne, Antipodes, 2007.

Pisano 2004: Giusy Pisano, Une archéologie du cinéma sonore, Paris, CNRS, 2004.

Rancière 2008 : Jacques Rancière, Le spectateur émancipé, Paris, La Fabrique, 2008. 